\title{
Erratum to: A fast DEA-based intra-coding algorithm for HEVC
}

\author{
Yingbiao Yao ${ }^{1}$ Tianjie $\mathrm{Jia}^{1} \cdot$ Xiaojuan $\mathrm{Li}^{1} \cdot \mathrm{Yu} \mathrm{Lu}{ }^{1}$
}

Published online: 13 April 2017

(C) Springer Science+Business Media New York 2017

\section{Erratum to: Multimed Tools Appl \\ DOI: $10.1007 / \mathbf{s 1 1 0 4 2 - 0 1 7 - 4 3 7 2 - Z ~}$}

The authors regret that acknowledgment of their funding source was omitted from the manuscript. The acknowledgment is written below:

Acknowledgments This work was supported by National Natural Science Foundation of China (61100044, 61671192), Zhejiang Province Science and Technology Innovation Focused Team Foundation (2013TD03).

The online version of the original article can be found at http://dx.doi.org/10.1007/s11042-017-4372-z

\section{Yingbiao Yao}

yaoyb@hdu.edu.cn

1 College of Communication Engineering, Hangzhou Dianzi University, Hangzhou Xiasha 2 Road, Zhejiang, Hangzhou 310018, China 